\title{
Mortalidad por enfermedad de Alzheimer en México de 1980 a 2014
}

\author{
Alfredo de Jesús Celis-De la Rosa, ${ }^{1}$ Carlos Enrique Cabrera-Pivaral, ${ }^{1}$ María Guadalupe Laura Báez-Báez, ${ }^{1}$ \\ Alfredo Celis-Orozco, ${ }^{1}$ Genaro Gabriel-Ortiz ${ }^{2}$ y Marco Antonio Zavala-González ${ }^{1}$ \\ ${ }^{1}$ Universidad de Guadalajara, Centro Universitario de Ciencias de la Salud, División de Disciplinas para el Desarrollo, Promoción y Preservación \\ de la Salud, Departamento de Salud Pública, Jalisco, México; ${ }^{2}$ nstituto Mexicano del Seguro Social, Centro de Investigación Biomédica de \\ Occidente, Jalisco, México
}

\begin{abstract}
Resumen
Introducción: Raramente se considera a las demencias como causa principal de muerte, por consiguiente existen pocos estudios sobre la mortalidad por Alzheimer a través de largos periodos. Objetivo: Describir la mortalidad por enfermedad de Alzheimer en México durante el periodo 1980-2014. Método: Estudio transversal en el que, con datos oficiales de mortalidad en México según los códigos 331.0 y G30 de la novena y décima revisiones de la Clasificación Estadística Internacional de Enfermedades y Problemas Relacionados con la Salud, se obtuvieron tasas crudas y estandarizadas de mortalidad por enfermedad de Alzheimer, nacional y por entidad federativa. Resultados: De ser casi inexistentes, en el quinquenio 2010-2014 se registraron tasas de 65.12 y 43.66 muertes por enfermedad de Alzheimer por cada 1000 mujeres y 1000 hombres, respectivamente. En todo el periodo estudiado, el grupo etario con las mayores tasas de mortalidad por esta causa fue el de mayores de 80 años, con 0.29 en 100000 habitantes durante 1980-1984 y 55.02 durante 2010-2014. La región con mayor mortalidad fue la noroeste, con tasas mayores a 2.28 en 100000 habitantes. Conclusiones: La mortalidad por enfermedad de Alzheimer es un problema de salud pública en México con tendencia creciente, especialmente entre mujeres y adultos mayores. Se requieren medidas diagnósticas precoces y tratamiento oportuno en primer nivel para aminorar este problema.
\end{abstract}

PALABRAS CLAVE: Enfermedad de Alzheimer. Mortalidad. México.

\begin{abstract}
Background: Dementias are rarely considered to be a main cause of death; therefore there are only few studies on Alzheimer's mortality covering long periods. Objective: To describe mortality from Alzheimer's disease in México for the period from 1980 to 2014. Method: Cross-sectional study where, with official mortality data in Mexico according to codes 331.0 and G30, respectively, of the $9^{\text {th }}$ and $10^{\text {th }}$ revisions of the International Statistical Classification of Diseases and Related Health Problems, crude and standardized Alzheimer's disease mortality rates were obtained, both nationally and by states. Results: From almost being inexistent, deaths from Alzheimer's disease went to a rate of 65.12 per 1000 females and 43.66 per 1000 males in the 2010-2014 five-year period. Throughout the study period, the age group with highest mortality rates for this cause were those older than 80 years, with 0.29 per 100,000 population in 1980-1984 and 55.02 in 100,000 in the 2010-2014 period. The region with the highest mortality was the northwest, with rates higher than 2.28 per 100,000 population. Conclusions: Mortality from Alzheimer's disease is a public health problem in Mexico with a growing trend, especially among women and older adults. Early diagnostic measures and opportune treatment are required in primary care in order to reduce this problem.
\end{abstract}

KEY WORDS: Alzheimer's disease. Mortality. Mexico.

Correspondencia:

Marco Antonio Zavala-González

E-mail: zgma_51083@yahoo.com.mx
Fecha de recepción: 08-03-2017

Fecha de aceptación: 08-03-2017

DOI: 10.24875/GMM.18003361
Gac Med Mex. 2018;154:550-554

Disponible en PubMed www.gacetamedicademexico.com 


\section{Introducción}

La enfermedad de Alzheimer es un trastorno neurológico incurable, progresivo y mortal de lenta evolución que afecta al cerebro humano, produciendo pérdida progresiva de la memoria, orientación, juicio y lenguaje ${ }^{1}$ que deriva en cambios de conducta y pérdida de la funcionalidad para las actividades cotidianas. $^{2}$ Es la causa más común de demencia, atribuyéndosele entre 60 y $70 \%$ de los casos, ${ }^{3}$ cuya prevalencia global se estima en $4.7 \%$ entre los individuos mayores de 60 años, lo que equivale a 35.6 millones de afectados, con una incidencia anual de 3.1 por cada 1000 personas de 60 a 64 años a 175 por cada 1000 personas de 95 años y más. ${ }^{4}$

En México se cuenta con información epidemiológica heterogénea sobre la frecuencia de demencias: la Encuesta Nacional de Salud y Envejecimiento en México reporta $3.3 \%$, y la Encuesta Nacional de Salud y Nutrición $7.9 \%$, en tanto se reconoce que los datos de esta última se equiparan a los de otros estudios. ${ }^{5}$ Se reconoce una incidencia anual de 27.3 por cada 1000 adultos mayores. ${ }^{6}$

Al margen de las cifras anteriores, es difícil evaluar la contribución independiente de las demencias a la mortalidad. En las actas de defunción raramente se les considera causa principal de muerte, ya que los afectados frecuentemente presentan otras comorbilidades que por sí solas pueden precipitar la muerte. ${ }^{4}$ Existen pocos estudios a escala estatal ${ }^{7}$ o nacional ${ }^{8,9}$ sobre mortalidad por Alzheimer y otras demencias en el transcurso de largos periodos de tiempo, ninguno en población mexicana, por consiguiente, la presente investigación se realizó con el objetivo de describir la mortalidad por enfermedad de Alzheimer en México a nivel nacional y por entidad federativa durante el periodo 1980-2014.

\section{Método}

Con base en estadísticas oficiales federales se llevó a cabo un estudio transversal en el que se estimaron las tasas de mortalidad por enfermedad de Alzheimer en México durante el periodo 1980-2014. Los numeradores, correspondientes al número de defunciones por enfermedad de Alzheimer, fueron obtenidos del Sistema Nacional de Información en Salud (Sinais) de México, ${ }^{10}$ considerando solo los registros con el diagnóstico de interés según los códigos de la novena y décima revisiones de la Clasificación Estadística
Internacional de Enfermedades (CIE-9, código 331.0 y CIE-10, código G30). ${ }^{11,12}$ En tanto que los denominadores, número de habitantes en México según edad, sexo y entidad federativa, se obtuvieron mediante proyección lineal a partir de las cifras poblacionales registradas en los Censos de Población y Vivienda de $1980,{ }^{13} 1990,{ }^{14} 2000^{15}$ y $2010^{16}$ y en la Encuesta Intercensal $2015,{ }^{17}$ Ilevados a cabo por el Instituto Nacional de Estadística y Geografía de México. Adicionalmente, se obtuvieron las tasas de mortalidad por demencias (CIE-9, códigos 290-294 ${ }^{11}$ y CIE-10, códigos F00-F03) ${ }^{12}$ durante el mismo periodo, para considerar posibles diagnósticos erróneos de la enfermedad de Alzheimer que pudieran condicionar subestimación o sobreestimación, en virtud de que independientemente de la etiología, comparten un cuadro clínico en común.

Se obtuvieron tasas de mortalidad estandarizadas según edad, sexo y entidad federativa por cada 100000 habitantes, de forma anual y quinquenal. La edad se clasificó en grupos según el criterio empleado por el Instituto Nacional de Estadística y Geografía: ${ }^{13-17}<1$ año, 1-4 años, 5-9 años, 10-19 años, 2039 años, 40-59 años, 60-69 años, 70-79 años y 80 años o más. Mientras que las tasas por entidad federativa se distribuyeron geoespacialmente por intervalos de clase obtenidos mediante la regla de Sturges. Este análisis se realizó con Epi-Info versión 7.1.5.2.

La investigación se consideró sin riesgo según la legislación nacional mexicana vigente al momento de realizar el estudio, dado que se emplearon métodos y técnicas de investigación documental retrospectivos y no se recopiló información nominal sensible de los individuos fallecidos. ${ }^{18}$

\section{Resultados}

En la Figura 1 se presentan las tasas de mortalidad estandarizadas anuales por enfermedad de Alzheimer durante el periodo de estudio, donde tanto en hombres como en mujeres se encontró una tendencia lineal ascendente en los fallecimientos por esta causa. En la Figura 2 se muestran estas tasas para otros diagnósticos de demencia durante el mismo periodo, con la misma tendencia, pero menos acusada.

En la Tabla 1 se exponen las tasas de mortalidad quinquenales durante el periodo de estudio, estratificadas según edad y sexo; se identificó un incremento de las tasas de mortalidad a mayor edad en ambos sexos, especialmente en las mujeres. 


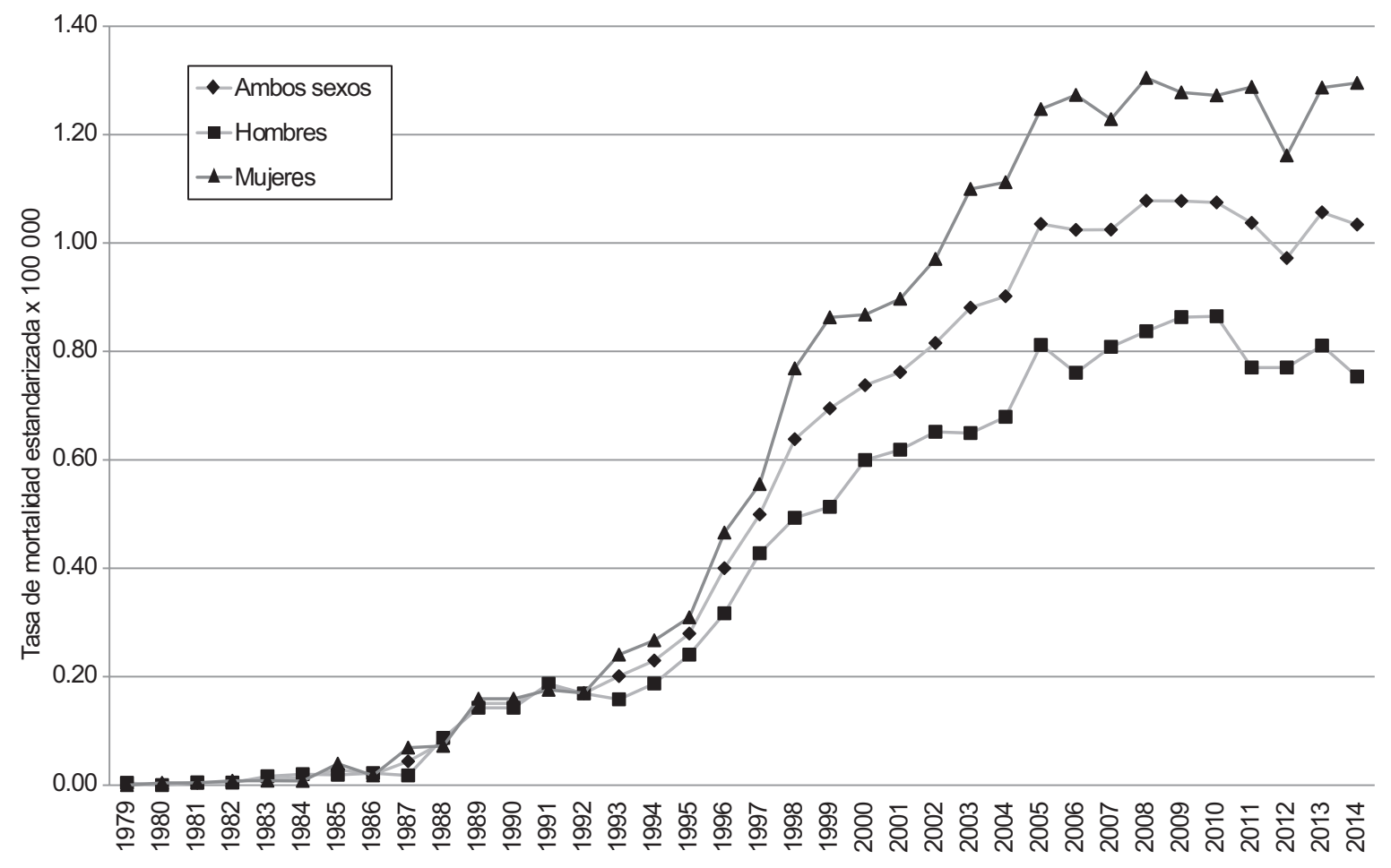

Aก̃o

Figura 1. Mortalidad anual con diagnóstico en el certificado de defunción de enfermedad de Alzheimer, México 1980-2014.

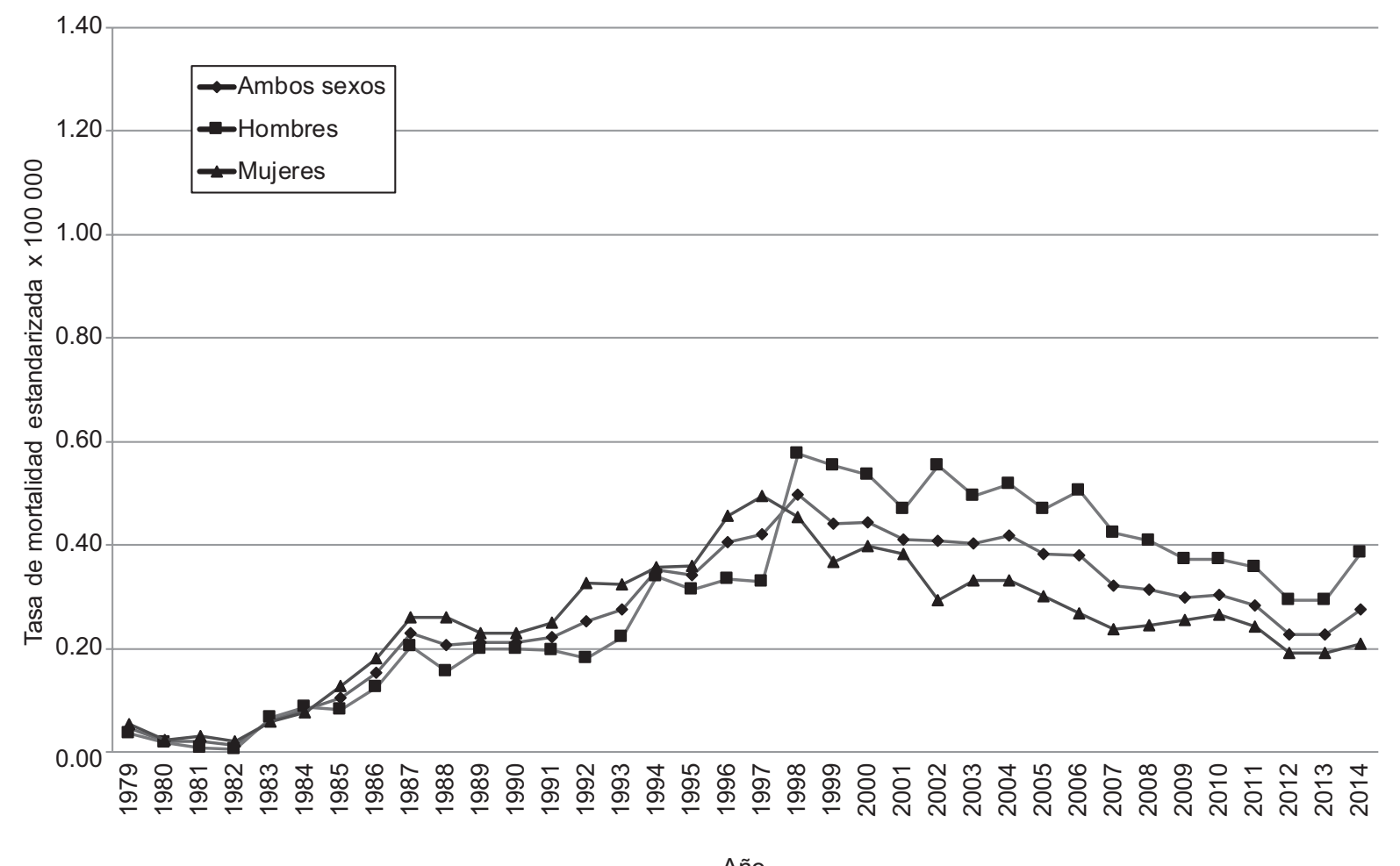

Figura 2. Mortalidad anual con diagnóstico en el certificado de defunción de demencia, México 1980-2014. 


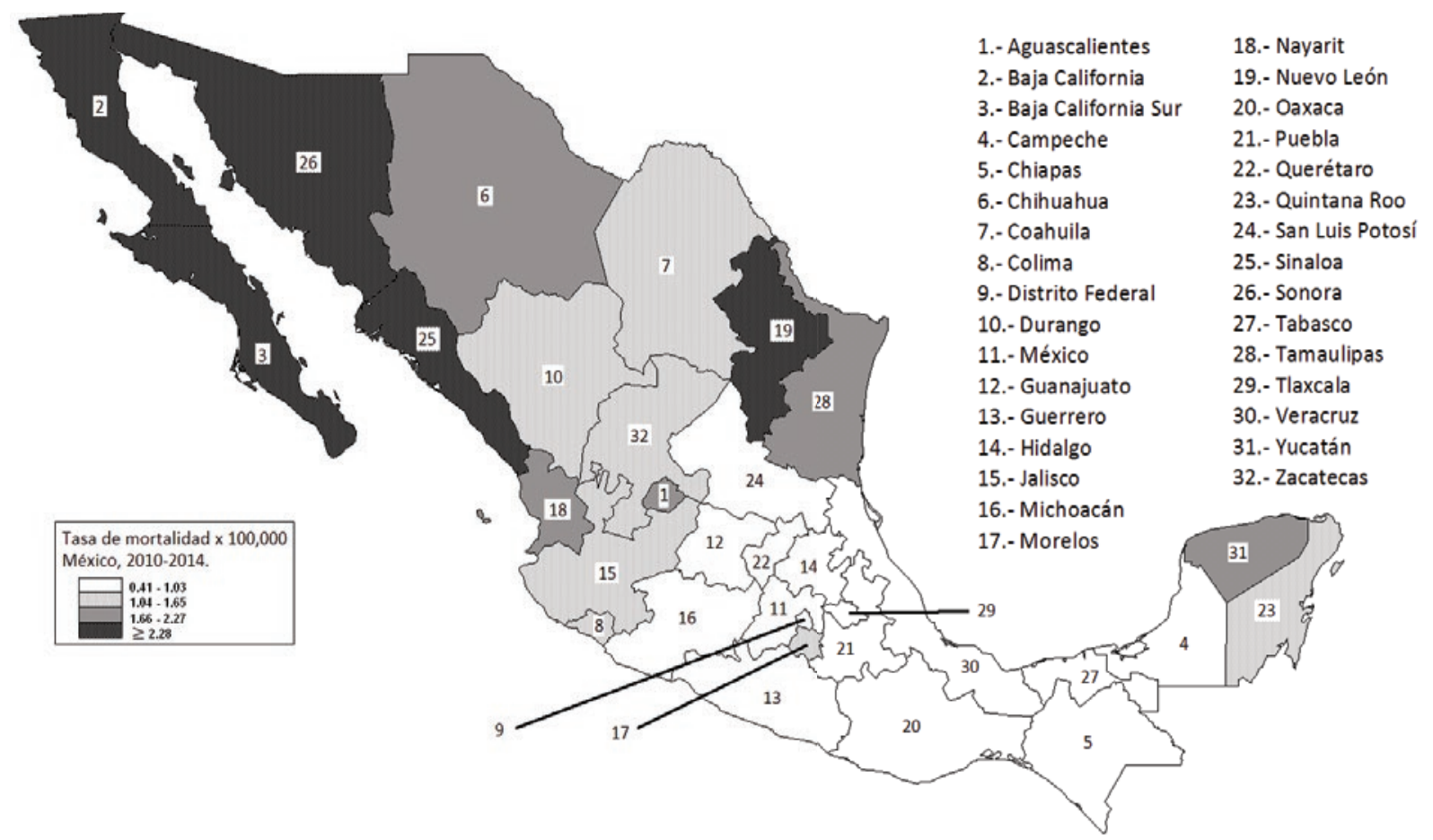

Figura 3. Mortalidad general por enfermedad de Alzheimer en México, 2010-2014, según entidad federativa.

Tabla 1. Mortalidad quinquenal por enfermedad de Alzheimer en México, 1980-2014, según edad y sexo

Grupos de edad Tasas estandarizadas $\times 100000$ habitantes (años) según quinquenio

1980-1984 1990-1994 2000-2004 2010-2014

Mujeres

40-59

$60-69$

$70-79$

$\geq 80$

0.00

\subsection{0}

0.13

0.12

Hombres

\begin{tabular}{lcccc}
$40-59$ & 0.01 & 0.08 & 0.12 & 0.11 \\
$60-69$ & 0.04 & 0.61 & 1.61 & 1.70 \\
$70-79$ & 0.03 & 2.76 & 9.52 & 10.03 \\
$\geq 80$ & 0.51 & 7.85 & 30.69 & 43.66 \\
& & & & \\
Población general & & & & \\
$40-59$ & 0.01 & 0.09 & 0.12 & 0.11 \\
$60-69$ & 0.03 & 0.64 & 1.73 & 1.69 \\
$70-79$ & 0.08 & 3.29 & 10.58 & 11.36 \\
$\geq 80$ & 0.29 & 7.53 & 39.52 & 55.02 \\
\hline
\end{tabular}

En la Figura 3 se muestran las tasas generales de mortalidad durante el quinquenio 2010-2014, estratificadas según entidad federativa. Las mayores tasas de mortalidad por enfermedad de Alzheimer se encontraron en Baja California, Baja California Sur, Nuevo León, Sinaloa y Sonora y las más bajas en el centro y sur de la República Mexicana (Morelos y Quintana Roo, respectivamente).

\section{Discusión}

Este es el primer estudio conocido por los autores en el que se describe la mortalidad por enfermedad de Alzheimer en México a nivel nacional y estatal, por el periodo de tiempo más amplio (35 años), que se suma a otras investigaciones similares en Andalucía, ${ }^{7}$ Inglaterra, ${ }^{8}$ Gales $^{8}$ y Estados Unidos. ${ }^{9}$

En consonancia con lo referido en los estudios citados $^{7-9}$ se observó una tendencia lineal ascendente en los decesos atribuidos a la enfermedad de Alzheimer, con predominio en el sexo femenino, crecimiento exponencial de las tasas de mortalidad a partir de los 60 años de edad y ocurrencia máxima en el grupo de 80 años y más en ambos sexos, lo que pudiera atribuirse al incremento en el diagnóstico de la enfermedad de Alzheimer durante las últimas dos décadas, distribución desigual de la enfermedad entre los sexos, momento de diagnóstico de la enfermedad, supervivencia desigual a la enfermedad según la comorbilidad, nivel de desarrollo de la población, aumento de la expectativa de vida al nacer en ambos sexos a partir de la década de 1940, superior en las mujeres respecto a los hombres, ${ }^{7-9}$ cuyo papel y contribución a estas diferencias deberán ser investigados posteriormente mediante estudios longitudinales. 
La tendencia de la mortalidad por enfermedad de Alzheimer se refleja a menor escala en las defunciones por otras demencias, lo que podría atribuirse al componente biológico de este grupo de enfermedades, que las hace más frecuentes en adultos mayores, cuyo número y longevidad se incrementa anualmente en México y el mundo, y que podría condicionar el aumento en el diagnóstico, certero o no, de estos padecimientos, lo que repercutiría en las estadísticas nacionales de morbilidad y mortalidad. En este tenor, la clasificación apropiada de estas enfermedades adquiere especial relevancia, pues es muy probable que una fracción significativa de quienes fallecieron por estas causas durante los 35 años del periodo estudiado hayan tenido otro tipo de demencia distinta a la registrada como motivo de defunción, como demencia vascular, con cuerpos de Lewy, alcohólica, frontotemporal o delirium. De ahí la importancia de la educación continua del personal médico en la materia.

Por otra parte, dado el continuo y acelerado avance de la medicina contemporánea es posible que eventualmente, a corto o mediano plazo, se identifiquen otros tipos de demencias, surjan nuevos criterios de diagnóstico o clasificación de las mismas o se formulen nuevas nomenclaturas, lo que también podría incrementar o disminuir las tasas de morbilidad y mortalidad atribuidas a estas, lo que implicaría un reinicio en la vigilancia epidemiológica de las demencias al imposibilitar la redefinición retrospectiva de los casos con base en nuevos criterios diagnósticos.

Independientemente de las limitaciones del presente estudio, inherentes a su diseño metodológico, los resultados refuerzan la necesidad de un plan de acción nacional contra la enfermedad de Alzheimer y otras demencias en México, dado que las presentes estadísticas reflejan un gran y creciente número de fallecimientos por estas causas, así como de su prevalencia, como lo señalan Gutiérrez-Robledo et al.5,6

El Plan de Acción Alzheimer y Otras Demencias México 2014, fruto del consenso de múltiples expertos nacionales, propone estrategias de prevención y promoción de la salud mental, el aseguramiento del acceso a servicios de salud de calidad, el diagnóstico y tratamiento oportunos de las personas afectadas por esta enfermedad, la formación de recursos humanos especializados suficientes, la promoción del respeto a los derechos humanos de las personas afectadas por esta enfermedad y sus cuidadores, el incremento de la investigación sobre esta y otras demencias, la evaluación periódica de la efectividad de las acciones y los cuidados a largo plazo en las últimas etapas de la enfermedad, ${ }^{5,6}$ que a la fecha del presente reporte han hecho poco eco y no se han visto materializadas en el sistema nacional de salud y a las que habría que agregar la prevención del encarnizamiento terapéutico, especialmente frecuente en la población geriátrica y cuyas motivaciones son complejas. ${ }^{19}$

Se puede concluir que pese a las reconocidas limitaciones de los estudios del presente tipo, ${ }^{7-9}$ donde se considera que las actas de defunción que alimentan las estadísticas oficiales pueden ser poco fiables, ${ }^{4}$ la creciente mortalidad observada por enfermedad de Alzheimer y otras demencias es un problema de salud pública en México que requiere atención inmediata, idealmente, mediante la implementación del plan de acción propuesto en años recientes. ${ }^{5,6}$

\section{Bibliografía}

1. Mestizo-Gutiérrez SL, Hernández-Aguilar ME, Rojas-Durán F, Manzo-Denes J, Aranda-Abreu GE. La enfermedad de Alzheimer y la diabetes mellitus. eNeurobiología. 2014;5(10):070914. Disponible en: https://www. uv.mx/eneurobiologia/vols/2014/10/Mestizo/Mestizo5(10)070914.pdf

2. Guía de práctica clínica. Diagnóstico y tratamiento de la demencia tipo Alzheimer. Evidencias y recomendaciones. México: Centro Nacional de Excelencia Tecnológica en Salud; 2010.

3. Demencia. Nota descriptiva. [Internet]. EE. UU.: Organización Mundial de la Salud; 2016. Disponible en http://bit.ly/1nqmcCa

4. Demencia: una prioridad de salud pública. EE. UU.: Organización Mundial de la Salud/Alzheimer's International; 2013.

5. Plan de acción Alzheimer y otras demencias. México: Instituto Nacional de Geriatría; 2014.

6. Gutiérrez-Robledo LM, Arrieta-Cruz I. Demencias en México: la necesidad de un Plan de Acción. Gac Med Mex. 2015;151(5):667-673. Disponible en: http://www.medigraphic.com/pdfs/gaceta/gm-2015/gm155p.pdf

7. Ruiz-Ramos M. Análisis de las principales variables determinantes de la evolución de la mortalidad por demencias en Andalucía. Rev Esp Salud Publica. 2012;86(3):219-228. Disponible en: http://scielo.isciii.es/scielo. php?script=sci_arttext\&pid=S1135-57272012000300002

8. Griffiths C, Rooney C. Trends in mortality from Alzheimer's disease, Parkinson's disease and dementia, England and Wales, 1979-2004. Health Stat Q. 2006;30:6-14.

9. Alzheimer's Association. 2016 Alzheimer's Disease facts and figures. Alzheimers Dement. 2016;12(4):459-509. DOI: 10.1016/j.jalz.2016.03.001

10. Sistema Nacional de Información en Salud. Mortalidad. [Internet]. México: Secretaría de Salud, 2015. Disponible en http://bit.ly/2jz6GXV

11. Clasificación Estadística Internacional de Enfermedades y Problemas Relacionados con la Salud. Novena revisión. Modificación clínica. EE. UU.: Organización Panamericana de la Salud/Organización Mundial de la Salud; 1977.

12. Clasificación Estadística Internacional de Enfermedades y Problemas Relacionados con la Salud, Décima revisión. EE. UU.: Organización Panamericana de la Salud/Organización Mundial de la Salud; 1992.

13. X Censo General de Población y Vivienda 1980. México: Instituto Nacional de Estadística y Geografía; 1981.

14. XI Censo General de Población y Vivienda 1990. México: Instituto Nacional de Estadística y Geografía; 1991.

15. XII Censo General de Población y Vivienda 2000. México: Instituto Nacional de Estadística y Geografía; 2001.

16. Censo de Población y Vivienda 2010. México: Instituto Nacional de Estadística y Geografía; 2011.

17. Encuesta Intercensal 2015. México: Instituto Nacional de Estadística y Geografía; 2016.

18. Reglamento de la Ley General de Salud en Materia de Investigación para la Salud. México: Diario Oficial de la Federación; 1986.

19. Covarrubias-Bermúdez MA, González-Huerta JI, Zavala-González MA. Obstinación terapéutica en pacientes geriátricos: estudio fenomenológico de experiencias de médicos en Jalisco, México. Medicina Paliativa. 2017;3:136-142. DOI: 10.1016/j.medipa.2016.10.002 